\title{
Ultra High Solid Angle EDS System Advanced STEM Analysis for FE-SEM
}

\author{
Y. Nakajima ${ }^{1}$, M. Suzuki ${ }^{2}$, S. Asahina ${ }^{1}$, N. Kikuchi ${ }^{1}$, K. Kawauchi ${ }^{1}$, M.Shibata ${ }^{3}$, N.Erdman ${ }^{3}$ and T. \\ Nokuo $^{1}$ \\ 1. JEOL.ltd, 3-1-2 Musashino, Akisima, Tokyo 196-8558 JAPAN \\ 2. Thermo Fisher Scientific K.K C-2F, 3-9 Moriya, Kanagawa, Yokohama 221-0022 JAPAN \\ 3. JEOL USA Inc. 11 Dearborn RD, Peabody, MA, 01960, USA
}

EDS (Energy dispersive X-ray spectrometry) is a technique used for elemental analysis of a sample, through detection of characteristic X-rays generated from a sample impacted by an electron beam. STEM (Scanning Transmission Electron Microscopy) is a method to obtain high spatial resolution images with Z-contrast [1]. STEM-in-SEM has recently become a technique of choice for high spatial resolution imaging in SEM of ultra-thin specimens and can be combined with the EDS for compositional analysis of nanostructures, with the ability to resolve structures previously unattainable with bulk EDS analysis.

There are two main ways to improve the spatial resolution of STEM-EDS on FE-SEM:

1. Increase the number of X-rays. This is difficult because of the thin sample size required for STEM-in-SEM imaging.

2. Optimization of detector solid angle and the detection of characteristic X-rays. There are technical difficulties producing the large detection area of EDS and constraints of choosing the correct distance between sample and EDS in general.

We have employed several strategies in order to improve EDS resolution in STEM-in-SEM mode. First, a large active area EDS system comprised of multiple EDS detectors [2] has been utilized for this analysis. Then, we further optimized the working distance of EDS detector with respect to the sample. We used two EDS detectors from Thermo Fisher Scientific having an active area of $60 \mathrm{~mm}^{2}$ and $100 \mathrm{~mm}^{2}$, giving a $160 \mathrm{~mm}^{2}$ active detection area in total. The latest model of JEOL FE-SEM JSM-7610F used in this study is equipped with a Schottky FE Gun to improve incidence of X-ray by high electron probe current, and features a semi in lens type objective lens to improve electron beam diameter.

Normally, the EDS detectors are located away from samples for safety reasons. We have safely set up shorter working distances resulting in a solid angle 5 times larger than previous conditions. Figure 1 shows STEM-EDS map on FE-SEM of DRAM that clearly shows around $10 \mathrm{~nm}$ size of oxidized layer even for 10 min mapping. It is possible to confirm the single $\mathrm{nm}$ Pt with 18 min EDS mapping shown in Figure 2 through the acquisition of the EDS map with high S/N ratio by using the ultra high solid angle EDS system on FE-SEM. This system not only performs STEM-EDS analysis but also high spatial resolution analysis on bulk samples with high efficiency. Additional analytical applications for various materials systems (bulk or ultra-thin specimens) using this setup will be discussed as well.

\section{References:}

[1] L. Reimer, Scanning Electron Microscopy: Physics of Image Formation and Microanalysis, second ed., Springer, Berlin, New York, 312-314, (1998)

[2] C. E. Lyman, et al,. High-performance X-ray detection in a new analytical electron microscope.

Journal of Microscopy. 176, 2, p 85-98, Nov. (1994) 

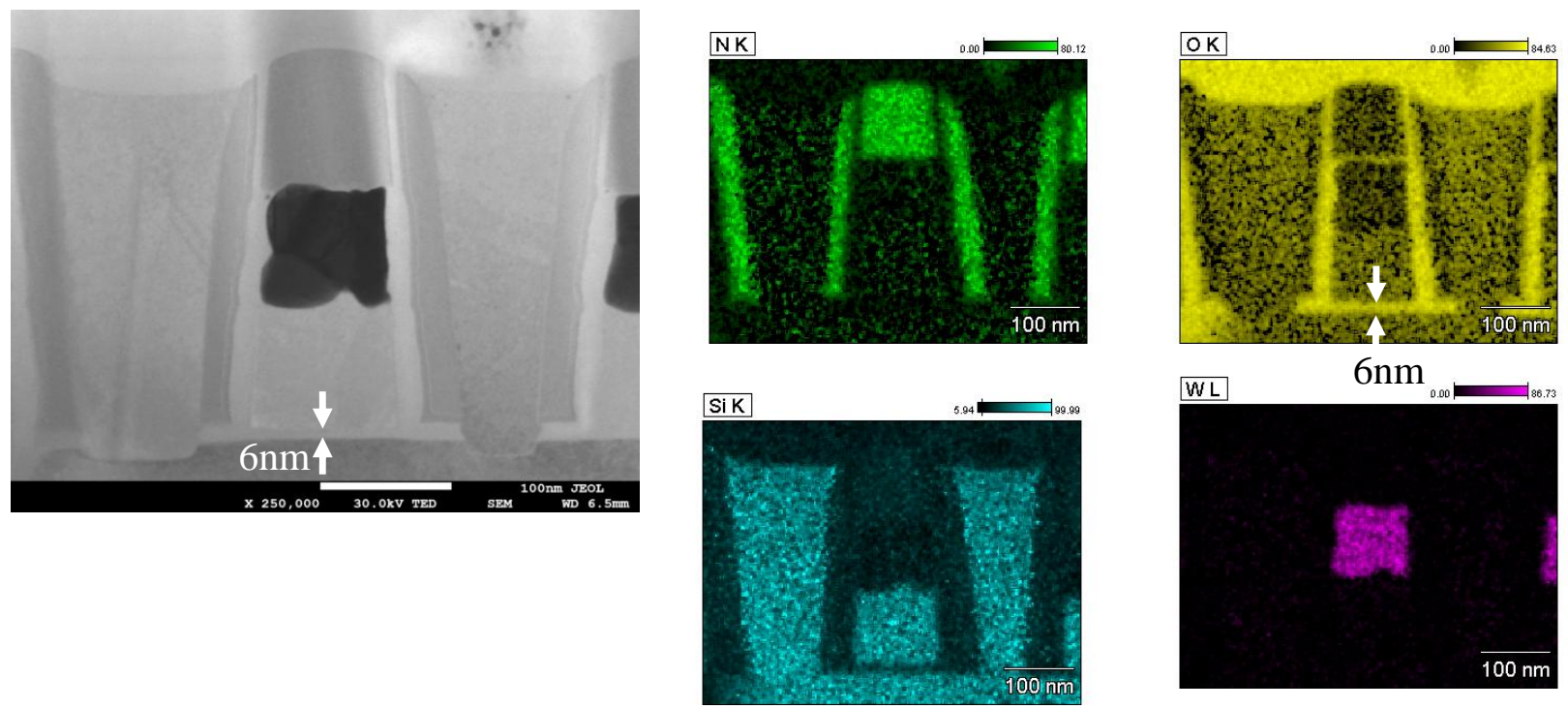

Figure.1. DRAM analysis by using ultra high solid angle EDS system
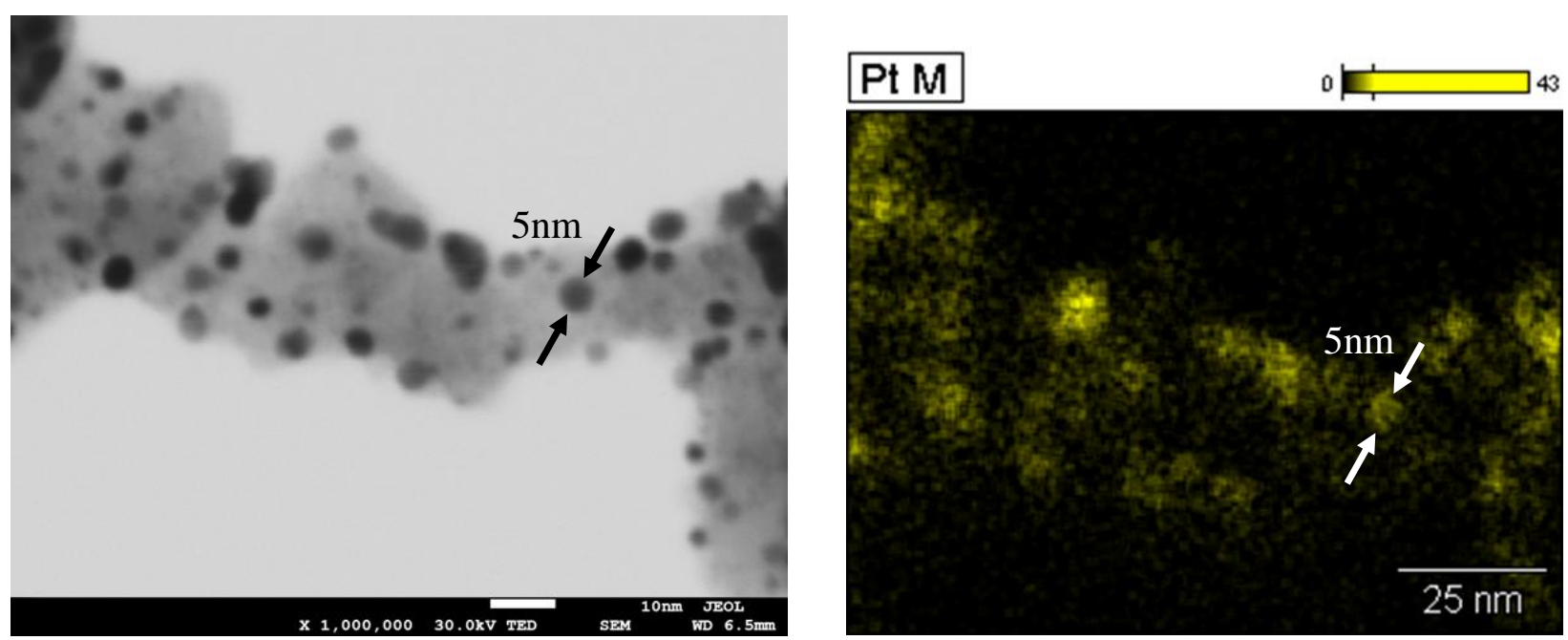

Figure 2. Pt on Carbon analysis by using ultra high solid angle EDS system 